\title{
Capacidades dinâmicas e rituais de interação entre alta e média gerência: proposta de um framework
}

\author{
Cristiano de Oliveira Maciel \\ Pontifícia Universidade Católica do Paraná \\ Kawana Harue Sato \\ Pontifícia Universidade Católica do Paraná \\ Heitor Takashi Kato \\ Pontifícia Universidade Católica do Paraná
}

O presente artigo foi desenvolvido considerando situações de incerteza ambiental, em que a ordem do dia ou questão estratégica (strategic issue) é a inovação dos recursos, ou seja, transformação, renovação ou desenvolvimento de uma nova capacidade funcional (ordinária). Com o objetivo de compreender como as capacidades dinâmicas desempenham esse papel na inovação dos ativos organizacionais em tais contextos ambientais, propõe-se aqui uma perspectiva alternativa de análise a partir de uma vertente microssociológica, a qual recorre aos elementos constitutivos de rituais de interação segundo Collins (2004). Essa perspectiva oferece possibilidades de investigação da influência das conversações estratégicas, que ocorrem em meio a práticas ritualísticas de interação entre alta e média gerência, sobre o potencial de inovação das capacidades organizacionais. Para compreensão da articulação teórica realizada no trabalho, apresenta-se um framework de análise que agrega a incerteza no nível macro com variáveis comportamentais e organizacionais no nível micro, enquanto elementos mais salientes dos rituais de interação. Visando estudos futuros e melhor compreensão do framework do trabalho, são apresentadas proposições acerca das relações do modelo após o detalhamento da estrutura analítica.

Palavras-chave: capacidades dinâmicas; rituais de interação; alta gerência; média gerência.

Dynamic capabilities and interaction rituals between top and middle managers: a framework proposal

This article was developed considering environmental uncertainty, in which the agenda or strategic issue (strategic issue) is the innovation of resources, i.e., upgrading, renovation or development of a new functional capacity (ordinary). The aim of this work is to develop a framework to encompass the role dynamic capabilities plays in the innovation of organizational assets in such environmental context. We propose an alternate perspective of analysis from a microsociological strand, which uses the

Artigo recebido em dez. 2010 e aceito em jul. 2011. 
interaction rituals components from Collins (2004). This perspective offers possibilities to investigate the influence of strategic talks on the organizational innovation capacity which take place amid ritual practices of interaction between top and middle managers of an organization. To create the theoretical articulation in the paper we present an analytical framework that brings the uncertainty on the macro variables and organizational behavior, at the micro level, as the most salient elements of ritual interaction. Aiming to stimulate further studies and better understanding, we present the propositions about the relations of the model after detailing the analytical framework.

KEY WORDs: dynamic capabilities; interaction rituals, top managers, middle managers.

\section{Introdução}

À medida que mudanças ambientais se tornam a regra em dado setor e não são mais percebidas como eventos isolados, é necessário que a estratégia também se reconfigure no nível macro, de um padrão na alocação de recursos para uma rotina na inovação dos ativos organizacionais, orientada tanto ao alcance de eficiência quanto de flexibilidade (Davis, Eisenhardt e Bingham, 2009; Lockett, Thompson e Morgenstern, 2009). No nível micro, entretanto, as ações que dão sustentação a esse processo são muito mais sutis (Sirmon, Hitt e Ireland, 2007) e, atualmente, apenas perifericamente abordadas pela Teoria Baseada em Recursos no que tange ao papel dos gerentes (Barney e Clark, 2007), como alertam Lockett, Thompson e Morgenstern (2009) e Sirmon e Hitt (2009).

Embora o papel dos atores organizacionais na exploração da (i) aplicação de novas funcionalidades, (ii) recombinação e (iii) criação de ativo tenha sido destacado há mais de 50 anos na obra de Penrose (1959), é recente o grupo de artigos que se propõe a examinar a relação entre aspectos comportamentais e atitudinais da gerência e a base de recursos. Como sugerem Sirmon, Hitt e Ireland (2007), é mínima a teoria destinada a explicar como gerentes transformam recursos. Em função da necessidade de avanços teóricos nessa direção, o objetivo do presente estudo é propor um framework para articular macro e microfundações das capacidades dinâmicas, a partir das interações entre alta e média gerência. Em relação aos aspectos macro, a proposta não tem a pretensão de avançar, pois se restringe à dimensão incerteza do ambiente externo das organizações.

Por outro lado, onde se assentam as principais contribuições do artigo, destaca-se que há impossibilidade de um mapeamento completo das capacidades dinâmicas, tanto em termos teóricos quanto empíricos. E, assim como outros fenômenos organizacionais, o avanço na compreensão sobre esse tema depende de novas propostas que possibilitem visualizar, mesmo que parcialmente, seus principais elementos constituintes, bem como as relações entre esses componentes. Ademais, o trabalho avança no sentido de olhar, como já apontado, para um aspecto até então negligenciado pela literatura, que é a noção de capacidades dinâmicas como atitudes e comportamentos gerenciais enquanto elementos recursivos - que formam e são formados - dos rituais de interação - por exemplo, reuniões, workshops e conversas informais - entre alta e média gerência. 
Em relação à estrutura de apresentação, o trabalho se divide nos seguintes tópicos principais após essa introdução: (i) capacidades dinâmicas: origens e conceitos; (ii) rituais de interação e ação gerencial; (iii) capacidades dinâmicas como rituais de interação; e (iv) framework de análise das capacidades dinâmicas.

\section{Capacidades dinâmicas: origens e conceitos}

A Teoria Baseada em Recursos explica como a vantagem competitiva é alcançada pelas organizações e como ela pode ser sustentada ao longo do tempo (Barney, 1996; Teece, Pisano e Shuen, 1997). Recursos desenvolvidos e controlados pelas empresas figuram como a principal fonte de vantagem competitiva. E, quando tais recursos são valiosos, raros, inimitáveis e insubstituíveis, a firma poderá alcançar vantagem competitiva sustentada, por meio da implementação de estratégias de criação de valor, não implementadas por outras organizações e não copiáveis pelos atuais e potenciais concorrentes. Assim, recursos homogêneos e móveis não podem ser considerados estratégicos (Barney, 1996).

Entretanto, a falta de esclarecimentos dessa teoria quanto aos métodos e razões de as firmas obterem vantagem competitiva em situações de mudanças rápidas e imprevisíveis levou pesquisadores ao desenvolvimento de uma nova perspectiva: a abordagem das capacidades dinâmicas, que figura, segundo alguns autores, como uma extensão da teoria baseada nos recursos (Eisenhardt e Martin, 2000; Teece, Pisano e Shuen, 1997).

Nessa linha de raciocínio, Eisenhardt e Martin (2000) afirmam que, em ambientes turbulentos, vantagens competitivas não são sustentáveis. De acordo com os autores, em tais ambientes o desempenho superior é decorrente da soma de vantagens temporárias, criadas continuamente. Essa habilidade em alcançar novas fontes de vantagem competitiva é denominada "capacidades dinâmicas". Dinâmico significa a capacidade de renovar competências diante de um ambiente de rápidas mudanças. O termo capacidades refere-se mais amplamente ao papel da gerência em adaptar, integrar e reconfigurar apropriadamente habilidades organizacionais internas e externas, recursos e competências funcionais, a fim de adaptar-se a um ambiente altamente mutável (Teece, Pisano e Shuen, 1997). Mais especificamente, Teece, Pisano e Shuen (1997) estabelecem três fatores responsáveis pela sustentação e evolução das capacidades dinâmicas: (i) processos gerenciais e organizacionais; (ii) posições ou qualidade dos ativos; e (iii) trajetórias (paths).

Processos gerenciais e organizacionais têm três funções principais: (i) coordenação/ integração (conceito estático); (ii) aprendizagem (conceito dinâmico); e (iii) reconfiguração (conceito transformacional). A coordenação/integração se dá sob uma racionalidade ou coerência para processos e sistemas, o que não equivale à ideia de cultura organizacional. A racionalidade subjacente à coordenação se reflete na noção de rotinas organizacionais. Essas rotinas podem impedir a inovação organizacional, visto serem baseadas em uma racionalidade que privilegia a eficiência, um tipo de aprendizagem exploitation em vez de exploration. A 
aprendizagem ocorre por meio de um processo social e coletivo, envolvendo tanto habilidades individuais quanto organizacionais, e requer códigos comuns de comunicação e procedimentos coordenados de exploração (busca). Ela possibilita, por meio da repetição e experimentação, executar processos de modo mais eficiente, melhor e radicalmente inovador. Por sua vez, a reconfiguração revela a habilidade de uma organização em responder rapidamente a mudanças ambientais. Essa capacidade da organização em transformar a si mesma representa também uma habilidade de aprendizagem.

Em relação à posição (endowments) dos ativos, Teece, Pisano e Schuen (1997) esclarecem que atributos da firma determinam sua postura estratégica. Esses ativos incluem: ativos tecnológicos protegidos por direitos de propriedade, ativos complementares, financeiros, de reputação, estrutura formal e informal, institucional (país ou região), posicionamento de mercado e limites organizacionais (por exemplo, grau de integração vertical).

No que concerne à dependência do caminho, os autores destacam que a posição atual dos ativos específicos da firma é resultado de sua trajetória passada, denotando que a história importa. Desse modo, as rotinas de uma organização condicionam seu comportamento futuro.

Mas, apesar de a conceitualização de Teece, Pisano e Shuen (1997) ser a pioneira e ter servido como base para trabalhos posteriores, entre as muitas definições encontradas na literatura para o termo capacidades dinâmicas, os pesquisadores que adotam essa perspectiva de análise tanto enfatizam aspectos comuns quanto características distintas, como se percebe nos conceitos apresentados a seguir:

v Habilidade em integrar, construir e reconfigurar competências internas e externas em resposta a rápidas mudanças ambientais (Teece, Pisano e Schuen, 1997:516).

- Processos para integrar, reconfigurar, obter e liberar recursos para se ajustar a (ou criar) mudanças de mercado. Assim, capacidades dinâmicas são as rotinas organizacionais e estratégicas por meio das quais firmas alcançam novas configurações de recursos, enquanto mercados emergem, colidem, dividem-se, evoluem e morrem (Eisenhardt e Martin, 2000:1107).

、 Um padrão estável e apreendido de atividade coletiva por meio da qual a organização sistematicamente gera e modifica suas rotinas operacionais em busca de melhorias em sua eficácia (Zollo e Winter, 2002:340).

Capacidades que operam para ampliar, modificar ou criar capacidades ordinárias (Winter, 2003:991).

- Habilidades para reconfigurar recursos e rotinas organizacionais no modo avaliado e considerado adequado por seus principais decisores (Zahra, Sapienza e Davidson, 2006:918).

v Capacidade de identificar e moldar oportunidades e ameaças, aproveitar as oportunidades e manter competitividade por meio do reforço, combinação, proteção. E, quando necessário, reconfiguração dos ativos tangíveis e intangíveis da organização (Teece, 2007:1319). 
จ Capacidades não imitáveis e particulares que uma organização possui para moldar e remodelar, configurar e reconfigurar ativos para responder a mudanças tecnológicas e de mercado e desviar de condições de retorno zero (Augier e Teece, 2008:1190).

Entre as características compartilhadas que são enfatizadas nessas diferentes definições estão: (i) mudanças ambientais; (ii) rotinas ou processos organizacionais; e (iii) capacidade de reconfiguração dos recursos. Mas, ainda que essas características tenham conduzido a um maior grau de consenso em relação ao conceito, é comum que existam sérias confusões na literatura a respeito do tema. A primeira delas reside na noção de termos contraditórios, que fazem da expressão um aparente oximoro. Isoladamente, o termo "capacidade" se refere a um processo organizacional. Assim, ele se configura como uma ação dividida em fases, que ocorre de forma contínua e tem sua eficiência aumentada ao longo do tempo por meio da repetição. Por outro lado, o termo "dinâmico" é frequentemente empregado para fazer alusão a tudo que um processo não é, ou seja, descontínuo e composto por mudanças. Desse modo, ao somar a reflexão isolada sobre um e depois o outro termo do conceito, "capacidades dinâmicas" acaba por não refletir a verdadeira essência da perspectiva inicial de Teece, Pisano e Shuen (1997).

Logo, uma capacidade dinâmica não pode ser tomada simplesmente como qualquer espécie de ativo conforme tantos outros citados na literatura da Teoria Baseada em Recursos. O conceito só pode ser mais amplamente compreendido ao considerar os dois termos em unidade, pois as capacidades dinâmicas se constituem em processos que impactam com os demais recursos, que servem para o desenvolvimento de uma base mais adequada de ativos organizacionais (Ambrosini e Bowman, 2009) e não como uma capacidade em contínua transformação. É preciso esclarecer que o que é dinâmico é a transformação causada por uma espécie de rotina sobre os recursos e não a capacidade em si, como a expressão "capacidades dinâmicas" parece sugerir.

Outros enganos aparecem até mesmo no trabalho de Teece (2007) sobre as microfundações das capacidades dinâmicas. $\mathrm{O}$ autor parece partir em direção a uma tentativa de demarcação de todo e qualquer elemento organizacional que possa impactar ou que constitui as capacidades dinâmicas. Nessa empreitada, o pesquisador peca substancialmente no quesito parcimônia em relação ao número de elementos a ser considerado na compreensão de seu framework, o que torna sua proposta não operacional. O autor considera tantos elementos em sua estrutura analítica (por exemplo, estrutura, processos de pesquisa e desenvolvimento, relações com fornecedores, processos de identificação de necessidades de mercado, processos decisórios, modelo de negócios, fidelidade e comprometimento, governança, gestão do conhecimento etc.) que o torna desinteressante para quem tenta operacionalizar o conceito de capacidades dinâmicas. Desse modo, contribui apenas para refrear as iniciativas de trabalhos empíricos a partir dessa perspectiva, que já são surpreendentemente reduzidos, conforme destacado por Ambrosini e Bowman (2009).

Mas talvez o maior dos problemas ainda presentes nessa abordagem se centre no aporte de teorias que não contribuem substancialmente para explicação das capacidades dinâmicas. 
É comum que pesquisadores adotem perspectivas econômicas e estratégicas no tratamento do tema, sem recorrer a conceitos de ordem social, política e cultural, que caracterizam mais fortemente a ideia de capacidades. Tal exercício tem impossibilitado, sobretudo, que se delimite mais precisamente o fenômeno a partir do que realmente interessa: processos e rotinas, conceitos tão caros a essa abordagem.

Até mesmo Teece (2007), pesquisador que cunhou o conceito de capacidades dinâmicas, incorre nessa limitação em sua proposta de apontar as microfundações das capacidades dinâmicas. Na apresentação de seu framework analítico, o autor esclarece nas conclusões do trabalho que recorreu principalmente a abordagens teóricas de disciplinas como: administração estratégica, economia industrial, história de negócios, estudos sobre inovação, leis e economia. É fundamental notar que, mesmo que Teece (2007) continue fiel à ideia de rotinas como o elemento nuclear do conceito de capacidades dinâmicas, ele não recorre a vertentes teóricas de natureza antropológica ou sociológica, o que limita sobremaneira a análise de aspectos micro, como propõe o pesquisador em seu artigo.

Ao destacar a influência da estrutura organizacional, por exemplo, como um dos aspectos nucleares das microfundações das capacidades dinâmicas, Teece (2007) permanece no nível macro ou meso de análise. O autor considera o papel da estrutura apenas em sua dimensão de arcabouço estrutural (papéis previamente definidos de quem se reporta a quem) e desconsidera a dimensão padrões de interação, que ocorre efetivamente na esfera micro (Ranson e Hinnings e Greenwood, 1980).

É importante lembrar que, além do arcabouço estrutural, existem padrões de interação entre os atores organizacionais que se conformam em rotinas com forte influência sobre os consequentes das capacidades dinâmicas - diferentes formas de inovação no uso dos recursos. Essas rotinas, que fogem à estrutura que pode ser representada tão linear e claramente em um organograma, contemplam uma sistemática que só pode ser mais bem apreendida ao considerar as interações entre os principais atores organizacionais no que concerne à formação da estratégia. Mais especificamente, essas interações de ordem estratégica envolvem alta (Top Management Team — TMT) e média gerência (Middle Management Team — MMT), o que permite propor a noção de "rituais de interação" (Goffman, 1967) organizacionais como elemento centralmente representativo do conceito de capacidades dinâmicas na esfera micro.

Visto que a habilidade de obter novas formas de vantagem competitiva é aspecto imprescindível em ambientes dinâmicos, a capacidade organizacional de aprendizado, a fim de alterar a configuração de recursos, diante de mudanças contextuais, torna-se crucial (Eisenhardt e Santos, 2006). O processo de aprendizado organizacional que ocorre por meio da incorporação de novas informações altera os padrões de comportamento anteriores e leva a resultados possivelmente melhores. A criação de novos conhecimentos por meio desse processo de aprendizagem, baseado em interações verticais e horizontais, forma a base do crescimento da organização por meio da recombinação dos recursos existentes da firma (Penrose, 1959) e pelo desenvolvimento e modificação das rotinas (Eisenhardt e Santos, 2006). 
Fundamentado principalmente nos conceitos de capacidades dinâmicas e de processo de aprendizagem organizacional (Eisenhardt e Santos, 2006), a Knowledge-Based View (KBV) também considera e reforça o papel do conhecimento como principal fonte de vantagem competitiva (Kogut e Zander, 1992) e, em termos estratégicos, como o mais importante dos recursos da firma (Grant, 1996).

Dessa perspectiva, as organizações passam a ser vistas como instituições de integração por meio de práticas ritualísticas, bases das ações coletivas e do processo de aprendizagem, uma vez que são capazes de criar condições culturais e materiais nas quais os indivíduos conseguem integrar seus conhecimentos individuais, bem como aqueles conhecimentos e informações compartilhados por diferentes grupos. Nesse sentido, o principal papel da organização reside na habilidade em criar um contexto favorável para integrar o conhecimento especializado individual (tácito e explícito) ao conhecimento de outros atores organizacionais, bem como aplicá-lo ao desenvolvimento de novas capacidades (Grant, 1996; Noda e Bower, 1996).

É importante destacar que o avanço do conhecimento via aprendizagem ocorre fundamentalmente a partir das interações entre os diferentes atores de uma organização. Essa interação se dá tanto na dimensão horizontal quanto na vertical, o que equivale a dizer: novos conhecimentos são criados tanto por meio de conversações e trocas de informações entre gerentes de um mesmo nível hierárquico quanto entre gerentes de níveis organizacionais distintos. Entretanto, há maior possibilidade de inovação nas interações entre gerentes de diferentes níveis hierárquicos, mesmo que esses mantenham laços mais fracos com gerentes de outro nível. Como destacam os pesquisadores da área de redes sociais, atores que fazem parte de um mesmo grupo acabam por deter um conhecimento mais redundante, e só por meio de laços fracos (contatos de menor frequência e menor intensidade emocional) e buracos estruturais (contatos não redundantes) é que as possibilidades de inovação no conhecimento são aumentadas (Burt, 1992; Wasserman e Faust, 1999).

Nesse sentido, os encontros entre atores organizacionais de diferentes níveis, como são as interações entre gerentes de alto e médio nível, figuram como aspecto central na constituição das capacidades dinâmicas. Desse modo, propõe-se no presente trabalho que os rituais de interação, por meio dos quais ocorrem as conversações estratégicas entre esses gerentes, formam o núcleo do conceito de capacidades dinâmicas.

Vale notar que colocar os rituais de interação entre alta e média gerência como elemento central na constituição das capacidades dinâmicas atende plenamente aos requisitos mais tradicionais do conceito desde sua primeira exposição em Teece, Pisano e Shuen (1997). Em primeiro lugar, esse elemento, ao mesmo tempo que esclarece a noção de capacidade gerencial coletiva, também melhor delimita quem faz parte dessa coletividade, demarcando as interações entre gerências de dois níveis, o que permite mais fácil operacionalização em comparação ao exame de todo e qualquer processo organizacional. Em segundo lugar, o padrão de interações entre gerentes é um ativo com posição (endowments) substancialmente diferenciada, específica a cada sistema organizacional. Em terceiro lugar, é preciso destacar que essas interações são socialmente construídas e, portanto, moldadas pelas dependências do caminho (path dependence). 
Essas características, que foram detalhadas inicialmente por Teece, Pisano e Schuen (1997), e que são atendidas pela concepção de capacidades dinâmicas como rituais de interação entre alta e média gerência, fazem desse tipo de ativo organizacional um recurso valioso, porque possibilita o compartilhamento e a combinação de conhecimentos distintos que estão nos níveis de indivíduo e de grupo, e, por consequência, contribui para diferentes modos de inovações (Dutton et al., 1997; Johnson, Neave e Pazderka, 2002). Ao mesmo tempo, esse tipo de ativo também é raro, uma vez que é dependente da favorabilidade do contexto (Dutton et al., 1997), ou seja, das condições culturais e materiais oferecidas pela alta gerência, que podem encorajar, ou não, as propostas de inovação da média gerência. Do mesmo modo, as especificidades e o grau de favorabilidade do contexto organizacional às opiniões, sugestões e iniciativas da média gerência fazem dessa rotina um recurso custoso de imitar.

\section{Rituais de interação e ação gerencial}

Neste tópico discute-se o conceito de rituais de interação e apresentam-se os componentes da ação gerencial que constituem e ao mesmo tempo são centralmente influenciados pelos encontros sociais. Mais detalhadamente, é preciso considerar que as interações entre gerentes ocorrem em meio a práticas organizacionais, modos sistemáticos de se fazer as coisas. São esses modos sistemáticos e regras socialmente definidas que constituem um ritual organizacional (Goss, 2008). De acordo com Collins (2004), os rituais de interação apresentam os seguintes componentes: (i) copresença situacional (sincronização física); (ii) foco interacional (inteligibilidade mútua acerca da questão a ser discutida); (iii) solidariedade social (sentido de obrigação); (iv) simbolismo (construção de símbolos que representam as atividades do grupo); e (v) energia emocional (sentido de efervescência coletiva e individual).

$\mathrm{Na}$ esfera organizacional, entre os rituais mais comuns com estas propriedades estão: apresentações, cafés da manhã e almoços de negócios, jantares, festas de confraternização, atividades de comitês e reuniões (Domenico e Phillips, 2009). Sobretudo as organizações, que operam em ambientes turbulentos e estão conscientes da necessidade de ampliar suas fontes de informação por meio da interação com a média gerência, de reuniões e conversas estratégicas em díades, e ocupam posição privilegiada na dinâmica da inovação de recursos (i.e., desenvolvimento de novas capacidades ordinárias) à luz dessa proposta.

Essas interações, que constituem os rituais, quando somadas aos recursos discursivos e simbólicos de reuniões, não só possibilitam a identificação de novas informações, ou mesmo mobilização de estratégias emergentes (Mariotto, 2003), mas também criam a "energia emocional" (Collins, 2004) necessária às iniciativas (uma das etapas do envolvimento) da média gerência para o desenvolvimento de novas capacidades.

As consequências dos rituais de interação sobre a ação gerencial perpassam a atitude dos atores organizacionais de alta e média gerência produzindo consistentes padrões de agên- 
cia. De acordo com Goffman (1967), as pessoas vivem em um mundo de encontros sociais face a face. Nesses encontros, há tendência de que o ator social seja fiel a uma linha de ação ou padrão comportamental.

Nessa linha de raciocínio, Westley (1990) sustenta que a exclusão da média gerência, durante o desenrolar do processo decisório estratégico de organizações burocráticas, tem consequências negativas não apenas sobre a energia emocional, mas também sobre o grau de envolvimento desses gerentes com questões estratégicas (strategic issues). Envolvimento nessas situações de interação gerencial significa: (i) identificar problemas e propor objetivos; (ii) gerar alternativas a problemas apresentados; (iii) avaliar alternativas apresentadas; (iv) desenvolver detalhes sobre as alternativas apresentadas; e (v) tomar as ações iniciais para mudança (por exemplo, inovação dos recursos) (Wooldridge e Floyd, 1989, 1990). Logo, a exclusão da média gerência na tomada de decisão é um dos principais fatores responsáveis pela limitação na geração de novas informações, propostas e iniciativas de transformação dos recursos organizacionais.

Em outro trabalho, Wooldridge e Floyd (1989) apontam as consequências da participação no processo decisório sobre o consenso. De acordo com esses autores, o consenso é uma variável que resulta da combinação entre inteligibilidade e comprometimento com a estratégia. Assim, a falta de inteligibilidade, ou não compreensão da média gerência em relação à estratégia da forma como é compreendida pela cúpula estratégica, faz com que seu comprometimento seja reduzido, pois, afinal, nessas condições, não há convergência sobre o que fazer. E a interação entre baixa inteligibilidade e baixo comprometimento é definida como falta de consenso (inteligibilidade $\times$ comprometimento).

Desse modo, os padrões atitudinais e comportamentais da média gerência, no que concerne ao seu potencial para contribuir com propostas (issueselling) e iniciativas de desenvolvimento de novas capacidades organizacionais, são condicionados, junto de outros fatores, pelos rituais de interação com a alta gerência.

Nesse sentido, a alta gerência pode contribuir com a construção de um contexto mais favorável para que a média gerência possa apresentar propostas e ações estratégicas de inovação dos ativos. Os fatores que caracterizam um contexto como desfavorável à apresentação de ideias e iniciativas frequentemente se centram no risco de o gerente médio macular sua imagem com a alta administração, medo das consequências negativas de uma proposta equivocada aos olhos da alta gerência, condições de downsizing, falta de prontidão a escutar, incerteza ambiental e uma cultura de exclusão e de penalidades para violação das normas preestabelecidas e tomadas como certas (Dutton et al., 1997).

É de responsabilidade então da alta gerência a favorabilidade contextual e a arquitetura relacional e material que deem provimento para o envolvimento, inteligibilidade, comprometimento e consenso da média gerência em relação à estratégia de inovação de ativos organizacionais (Dutton et al., 1997), para que, de fato, as capacidades dinâmicas de uma organização possam operar, por meio dos rituais de interação, no desenvolvimento de novas capacidades ordinárias. 


\section{Capacidades dinâmicas como rituais de interação}

Como já indicado, a conceituação das capacidades dinâmicas como ativos constituídos centralmente a partir das interações entre alta e média gerência em conversações estratégicas, para proposições e exercício de reconfiguração da base de recursos, delimita mais especificamente um aspecto crucial no desenvolvimento de novas capacidades: processos coletivos de aprendizagem organizacional que contribuem acentuadamente para a síntese de conhecimentos de fontes diferenciadas em algo novo (Wang e Ahmed, 2007).

Essa definição de capacidades dinâmicas como rituais de interação gerencial torna mais explícitos suas variáveis e componentes principais. Como destacam Wang e Ahmed (2007), essas capacidades são construídas ao longo do tempo por meio de complexas interações entre os recursos da firma. A definição oferecida aqui converge com esse apontamento dos autores, mas vai além: estabelece-se na tentativa de delimitar quais variáveis e componentes de natureza social são fundamentais nesse tipo de rotina organizacional.

A importância do aspecto social, sobretudo no nível micro, tem sido surpreendentemente ignorada nessa literatura, apesar de diversos autores destacarem a importância de fatores de natureza social, cultural ou política nos processos organizacionais. Entre os fatores mais comuns estão: padrões comportamentais gerenciais que se conformam em rotinas organizacionais, relações de liderança, processos coletivos de aprendizagem estratégica, imersão social (embeddedness), comprometimento, envolvimento e consenso dos gerentes (Ambrosini e Bowman, 2009; Wang e Ahmed, 2007).

Atentar para esses fatores, especialmente aqueles que envolvem mais diretamente as relações e interações gerenciais, conduz a olhar para dentro da "caixa preta" da competência dos atores organizacionais em transformar recursos para criar valor em ambientes turbulentos (Sirmon, Hitt e Ireland, 2007). E destacar mais precisamente o papel da gerência média (Middle Management Team) em tal processo, de natureza predominantemente coletiva, significa estar em consonância com os principais pressupostos da literatura das capacidades dinâmicas. Essas capacidades são construídas pelos atores da firma e, portanto, não podem ser compradas no mercado, porque são dependentes do caminho e estão imersas em relações sociais (Ambrosini e Bowman, 2009).

Mais detalhadamente, essas interações gerenciais se dão por meio de rituais de conversação estratégica, formais ou informais (Goffman, 1967), quer seja em díades ou entre grupos. Os rituais de interação gerencial são blocos fundamentais da estratégia no plano micro. No plano macro, a estratégia pode ser vista como um padrão na alocação de ativos. Contudo, no plano micro ela toma forma a partir de rotinas baseadas centralmente em conversações entre membros da alta e média gerência para transformação da base de recursos em face a mudanças nas características contextuais. Como propõe Westley (1990), as negociações e interpretações entre gerentes de alto e médio nível que estão envolvidas na construção de sentido é um fator crucial da aprendizagem organizacional.

Os rituais de interação entre alta e média gerência revelam as condições da microdinâmica de inclusão presente em dada organização e tornam claro que o processo estratégico 
que envolve a alocação e transformação de ativos não é controlado estritamente por diretores e CEOs. Isso significa, mais precisamente, a possibilidade de especificar em que grau a cúpula estratégica dá espaços para sugestões, propostas e realizações da média gerência no que concerne à inovação nos modos de recombinação de recursos.

Mas vale destacar que mesmo que essa reconceituação, ou melhor, reespecificação do conceito pareça ser diametralmente oposta às definições indicadas em trabalhos anteriores, na verdade ela não destoa dos atributos considerados pela maioria dos pesquisadores dessa temática (por exemplo, Teece, Pisano e Schuen, 1997; Teece, 2007; Eisenhardt e Martin, 2000; Ambrosini e Bowman, 2009). Ao contrário: a presente proposta de reespecificação do conceito de capacidades dinâmicas como rituais de interação entre gerentes conserva os critérios de caracterização de Teece, Pisano e Shuen (1997), como já detalhado, e ainda apresenta algumas vantagens adicionais.

A primeira dessas vantagens é que o conceito destaca a característica de rotina, mais especificamente, por meio da lógica de rituais. Esses rituais se constituem em interações que são mais sistemáticas no que concerne à discussão de questões estratégicas (strategic issues) entre gerentes de alto e médio nível, em vez de se basearem em soluções esporádicas de problemas ad hoc. Da mesma maneira, essa definição não deixa de especificar em que medida as capacidades dinâmicas representam de fato um fenômeno coletivo. De acordo com a presente proposta, o esforço coletivo de reconfiguração da base de recursos ocorre tanto por meio das interações das díades quanto em eventos ou episódios estratégicos, como reuniões com a presença de gerentes de diferentes níveis. Por consequência, esse conceito contribui para que o pesquisador evite a tentação de localizar equivocadamente as capacidades dinâmicas apenas em uma pequena parte da organização (i.e., no setor de pesquisa e desenvolvimento) (Helfat, 1997).

Outra vantagem em colocar os rituais de interação entre gerentes como núcleo duro do conceito de capacidades dinâmicas se centra na possibilidade de melhor discriminação desse tipo de capacidade em relação a outros ativos (por exemplo, capacidades funcionais ou ordinárias). A caracterização das capacidades dinâmicas como um padrão estável de aprendizagem decorrente da integração de conhecimentos individuais e grupais de diferentes níveis também é um atributo positivo da proposta aqui apresentada.

Sobretudo, o aporte de uma perspectiva microssociológica (Collins, 2004), que permita destacar o fenômeno dos rituais de interação entre diferentes gerentes, coloca o conceito de rotina sob lentes mais acuradas em comparação com a perspectiva de Teece, Pisano e Shuen (1997). A delimitação das capacidades dinâmicas como um conceito coletivo e essencialmente social o faz mais operacional em termos empíricos. De acordo com Ambrosini e Bowman (2009), o fenômeno capacidades dinâmicas tem sido pobremente especificado, e assim impossibilitando que os pesquisadores que adotam tal perspectiva saibam o que olhar empiricamente (Ambrosini e Bowman, 2009). Por consequência, essa redefinição também contribui mais fortemente para a realização de estudos qualitativos, que compõem um número ainda muito reduzido (Ambrosini e Bowman, 2009). 


\section{Framework de análise das capacidades dinâmicas}

Macro e microfundações das capacidades dinâmicas, enquanto rituais de interação, são aqui articuladas por meio de um framework de análise. O modelo detalha os fatores que inibem e capacitam (Ambrosini e Bowman, 2009) as capacidades dinâmicas, contempla o papel do ambiente, mais especificamente da dimensão incerteza, bem como aborda os papéis da alta e da média gerência nas conversações estratégicas que envolvem questões (strategic issues) de transformação da base de recursos, como apresentado anteriormente.

A representação gráfica do modelo de análise proposto aparece na figura a seguir com a indicação das proposições (P1 a P13). De acordo com a figura, a incerteza é a principal dimensão do ambiente que está associada às capacidades dinâmicas e também relacionada a variáveis atitudinais e comportamentais (inteligibilidade, comprometimento, consenso, envolvimento) da média gerência que constituem e formam os rituais de interação.

A favorabilidade do contexto a questões de transformação de recursos, que são propostas pela média gerência, representa o contexto social, cultural e material proporcionado pelos gerentes da cúpula estratégica. As características ou propriedades desse contexto também são influenciadas pela dimensão incerteza do ambiente externo das organizações. A inovação no uso dos recursos representa a capacidade ordinária desenvolvida, em termos de frequência, importância ou aplicabilidade. As setas representam relações causais com valor positivo ou negativo. E a linha tracejada aberta faz lembrar que os rituais de interação entre alta (TMT) e média gerência (MMT) são apenas parte de um fenômeno mais complexo e imerso em outros processos organizacionais não contemplados nessa representação alternativa das capacidades dinâmicas.

Como se percebe, a ênfase do modelo reside na responsabilidade dos gerentes de nível médio e alto pelo "funcionamento" das capacidades dinâmicas. Esse recorte é justificado pelo fato de que, nos níveis inferiores a esses, práticas dadas como estratégicas adquirem status e importância essencialmente operacional e são, então, percebidas como capacidades ordinárias. Por isso, os limites para mapeamento das capacidades dinâmicas são definidos nesses dois níveis mais gerais.

Os rituais de interação (Collins, 2004) entre alta e média gerência, que são contemplados na estrutura analítica antes exposta, se situam nas questões estratégicas (strategic issues) envolvidas na transformação e recombinação de recursos - foco das capacidades dinâmicas. De acordo com o framework proposto, as interações gerenciais que ocorrem por meio de conversas estratégicas e que envolvem esse tema dependem do grau de incerteza ambiental que é percebido pelos gerentes. 
Figura

Capacidades dinâmicas como rituais de interação entre alta e média gerência

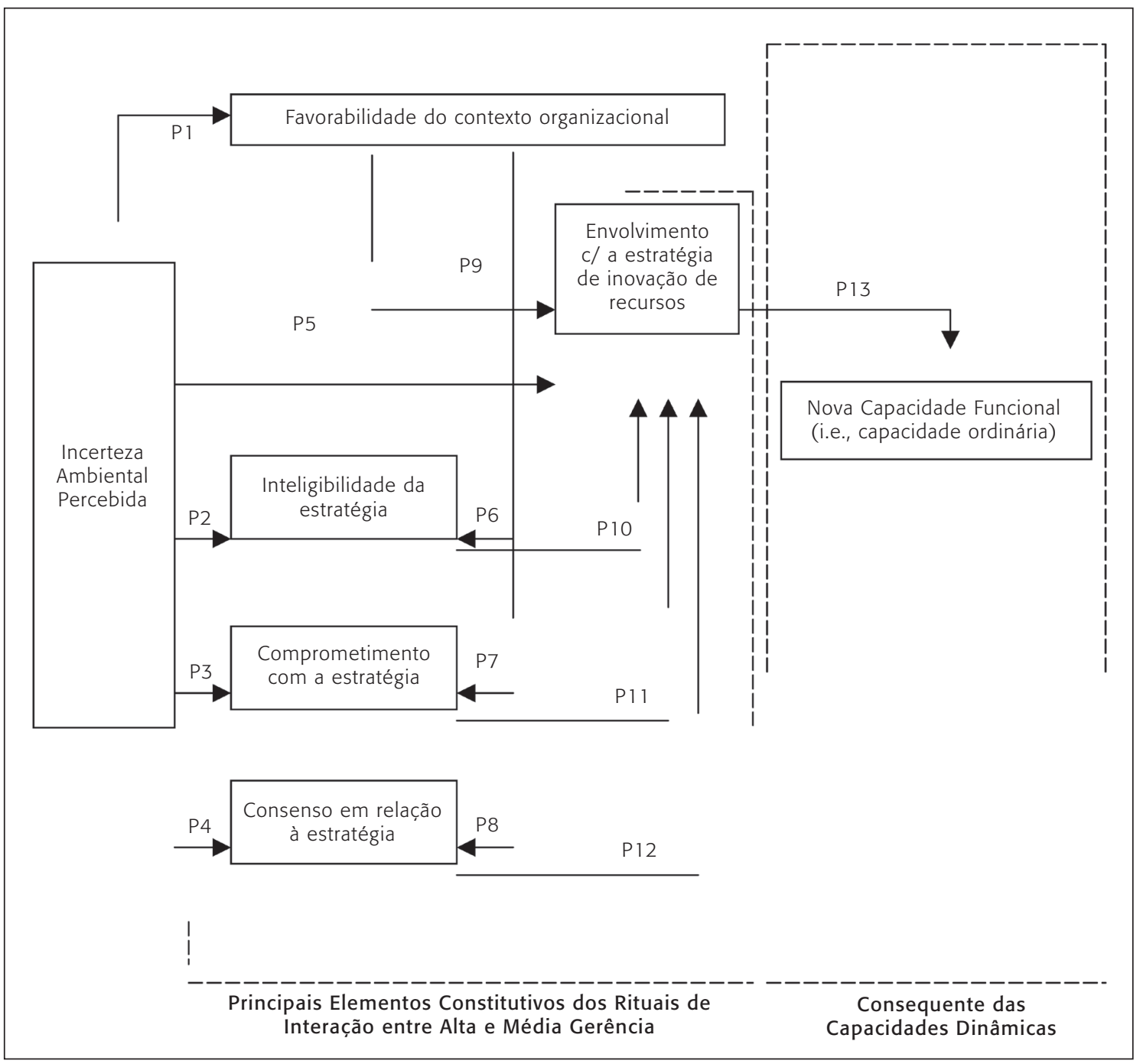

Os objetos da incerteza ambiental percebida variam entre muitos aspectos. Miles e Snow (2003), por exemplo, relacionam os seguintes elementos: fornecimento de insumos, ações dos competidores, demandas dos consumidores, disponibilidade de crédito e outras características do mercado financeiro, agências regulatórias e ações dos sindicatos. Mas, independente da dimensão na qual o grau de incerteza é percebido, todas demandam a mesma necessidade: geração de ideias e, consequentemente, de ações para absorção da incerteza (Thompson, 1967). No nível da alta gerência de organizações inseridas em ambientes mais imprevisíveis, o principal decisor organizacional e sua diretoria perceberão maior necessidade em fazer com 
que exista um clima mais favorável (Dutton et al., 1997), recompensador e livre de sanções, para exposição das ideias da média gerência, bem como estarão abertos a negociações e interpretações entre os gerentes de níveis iguais ou distintos. De tal assertiva foi desenvolvida a seguinte proposição:

Proposição 1: Quanto maior a incerteza ambiental percebida pela alta gerência maior será a favorabilidade do contexto organizacional ao envolvimento da média gerência com a estratégica de inovação de recursos.

Em termos globais de possibilidades de geração de novas ideias e ações de recombinação de recursos para construção de novas capacidades, a incerteza, no caso de organizações em ambientes turbulentos, pode impor necessidades que serão benéficas à alta administração. Entretanto, a incerteza ambiental não exerce os mesmos efeitos sobre a média gerência. A incerteza torna mais difícil que gerentes de nível médio compreendam a necessidade da estratégia de inovação de recursos e o foco interacional exigido por essa estratégia. E essa falta de foco interacional nos momentos de copresença situacional opera no sentido de reduzir a solidariedade social (obrigação social) desses atores (Collins, 2004; Goffman, 1967). Desse modo, ocorrem efeitos deletérios sobre a capacidade da gerência média em se comprometer com a estratégia (ter uma atitude favorável à estratégia, bem como ações estáveis e congruentes), uma vez que a estratégia não tenha sido adequadamente compreendida (Wooldridge e Floyd, 1990). Assim:

Proposição 2: Quanto maior a incerteza ambiental, menor será a inteligibilidade da média gerência em relação à estratégia de inovação de recursos.

Proposição 3: Quanto maior a incerteza ambiental menor será o comprometimento da média gerência em relação à estratégia de inovação de recursos.

Mas talvez o pior dos efeitos da incerteza sobre os aspectos comportamentais resida mais especificamente no consenso (Wooldridge e Floyd, 1989) em relação à estratégia de inovação de ativos. O consenso em relação à estratégia é definido como resultado da interação entre inteligibilidade (foco interacional) e comprometimento (solidariedade social) (Collins, 2004), logo:

Proposição 4: Quanto maior a incerteza ambiental, menor será o consenso da média gerência em relação à estratégia de inovação de recursos.

Outra variável comportamental da média gerência que é influenciada pela incerteza ambiental é seu envolvimento em conversações estratégicas. Esse envolvimento, para Wooldridge e Floyd (1990), ocorre por meio da detecção de problemas, apresentação de soluções, participação no processo de avaliação de possíveis alternativas para solução de problemas, 
elaboração de linhas de ação e iniciativas (ações) de mudança. A incerteza ambiental atenua o foco interacional, a solidariedade social e, principalmente, o simbolismo (construção de símbolos compartilhados para efetivamente decretação da noção de grupo) (Collins, 2004). Decorre daí que, em virtude de um maior grau de incerteza ambiental, o envolvimento da média gerência com a estratégia de inovação de ativos deve ser menor (Dutton et al., 1997). Daí estruturou-se a próxima proposição:

Proposição 5: Quanto maior a incerteza ambiental percebida pela média gerência, menor será seu envolvimento com a estratégia de inovação de recursos.

Mas mesmo que a incerteza ambiental apresente efeitos negativos sobre a inteligibilidade (foco interacional) (Collins, 2004), sobre o comprometimento, consenso e envolvimento da média gerência com a estratégia de reconfiguração da base de recursos, a cúpula organizacional (por exemplo, presidente e diretores) tem capacidade para contribuir com o desenvolvimento da energia emocional da gerência média e com a estruturação de um clima mais favorável a propostas de novas ideias (Dutton et al., 1997). Como já detalhado, a definição de favorabilidade do contexto se caracteriza pelos aspectos culturais e materiais que são oferecidos pela alta gerência, que potencializam a probabilidade de apresentação de propostas de inovação pela média gerência. Logo, a favorabilidade contextual influenciará positivamente os aspectos comportamentais e atitudinais de gerentes de nível médio:

Proposição 6: Quanto maior a favorabilidade do contexto organizacional, maior a inteligibilidade da estratégia de inovação de recursos para a média gerência.

Proposição 7: Quanto maior a favorabilidade do contexto organizacional, maior será o comprometimento da média gerência em relação à estratégia de inovação de recursos.

Proposição 8: Quanto maior a favorabilidade do contexto organizacional, maior será o consenso da média gerência em relação à estratégia de inovação de recursos.

Proposição 9: Quanto maior a favorabilidade do contexto organizacional, maior será o envolvimento da média gerência em relação à estratégia de inovação de recursos.

A preocupação com a associação da incerteza e do contexto organizacional proporcionado pela alta gerência em relação ao comportamento de gerentes de nível médio assenta-se na influência dessas variáveis sobre a inovação dos ativos organizacionais — desenvolvimento de uma nova capacidade. Pois, gerentes de nível médio com maior inteligibilidade (foco interacional) (Collins, 2004) acerca da estratégia de inovação de recursos, que se faz necessária em ambientes dinâmicos, e também mais comprometidos com a estratégia (solidariedade social), apresentam, por consequência, um maior consenso em relação à ação organizacional. E inteligibilidade, comprometimento, consenso e envolvimento, que se caracterizam como 
dimensões das práticas ritualísticas dos gerentes com a estratégia, são aspectos cruciais para o envolvimento com os planos e ações de renovação e transformação dos ativos organizacionais. Desse modo, são estabelecidas as seguintes proposições do modelo:

Proposição 10: Quanto maior a inteligibilidade da média gerência em relação à estratégia de inovação de recursos, maior será seu envolvimento com essa estratégia.

Proposição 11: Quanto maior o comprometimento da média gerência em relação à estratégia de inovação de recursos, maior será seu envolvimento com essa estratégia.

Proposição 12: Quanto maior o consenso da média gerência em relação à estratégia de inovação de recursos, maior será seu envolvimento com essa estratégia.

De acordo com o modelo proposto, o envolvimento da média gerência com a estratégia de inovação de recursos opera como elemento fundamental da efetiva construção e reconfiguração dos ativos organizacionais. Isso se deve à sequência de atividades que é prevista teoricamente na caracterização do envolvimento gerencial com esse tipo de estratégia: (i) identificar problemas e propor objetivos; (ii) gerar alternativas a problemas apresentados; (iii) avaliar alternativas apresentadas; (iv) desenvolver detalhes sobre as alternativas apresentadas; e (v) tomar as ações iniciais para mudança (por exemplo, reconfiguração dos recursos), como se extrai de Wooldridge e Floyd (1989, 1990). Assim, a última etapa das atividades que caracterizam o envolvimento da média gerência na estratégia de transformação de recursos centra-se na iniciativa para mudanças, a qual contribui, sobretudo, para a criação de uma nova capacidade organizacional ordinária. Então se formula a última proposição do modelo de capacidades dinâmicas, o qual está baseado nos elementos constitutivos dos rituais de interação:

Proposição 13: Quanto maior o envolvimento da média gerência com a estratégia de inovação de recursos, maior será a possibilidade de criação de uma nova capacidade ordinária (funcional).

Em resumo: o conjunto das proposições acima, que dá forma ao framework de análise proposto, destaca a relação entre incerteza ambiental, favorabilidade do contexto e aspectos comportamentais da média gerência em meio aos rituais de interação organizacional (Collins, 2004; Goss, 2008; Goffman, 1967). À luz de um pressuposto de realidade culturalmente sustentada, a dinâmica desses rituais representa em grande medida o conjunto de valores gerenciais que condicionam o modo como os principais decisores conduzem estrategicamente determinado empreendimento (Barney, 1986).

Em adição, deve ser destacado que esses elementos são considerados os principais imperativos constituintes da noção de capacidades dinâmicas numa perspectiva microssociológica (Collins, 2004; Goss, 2008; Goffman, 1967), a qual está voltada para a explicação das condições necessárias à renovação e ao desenvolvimento de capacidades ordinárias, e, por consequência, reconstrução das fontes de vantagem competitiva. 
Como resultado da proposta aqui apresentada, o conceito de capacidades dinâmicas é redefinido a partir de um ponto de vista micro, centrado principalmente nas conexões verticais entre gerentes organizacionais. Nesses termos, capacidades dinâmicas são processos organizacionais com dimensões atitudinais e comportamentais que impactam a habilidade de uma organização em renovar, reconfigurar e desenvolver novas capacidades ordinárias a partir de conversações estratégicas (Goffman, 1967) que ocorrem centralmente por meio de rituais de interação (Collins, 2004) entre alta (TMT) e média gerência (MMT).

Vale ressaltar que a inexistência ou fraca interação (sincronização física) (Collins, 2004) gerencial vertical não elimina a possibilidade de desenvolvimento das capacidades dinâmicas. Como já colocado, o framework acima não tem a pretensão de cobrir em sua plenitude todos os tipos de capacidades dinâmicas. Defende-se aqui apenas que a ausência dessa dinâmica ritualística (Collins, 2004; Goss, 2008; Goffman, 1967) limita sobremaneira a possibilidade de desenvolvimento de novas capacidades ordinárias.

\section{Considerações finais}

Como afirma Helfat (1997), é necessário aprender mais sobre como identificar e promover oportunidades para o compartilhamento de informações entre diferentes partes das organizações para desenvolvimento das capacidades dinâmicas. Congruente com essa preocupação da autora, o objetivo no presente trabalho foi propor uma estrutura analítica para articulação das macro e microfundações das capacidades dinâmicas, considerando centralmente o papel dos rituais de interação (Collins, 2004; Goss, 2008; Goffman, 1967) entre gerentes de alto e médio nível.

O modelo apresentado destacou na esfera macro a dimensão ambiental incerteza e no nível micro as seguintes variáveis comportamentais da gerência média: inteligibilidade, comprometimento e consenso em relação à estratégia, que foram somados ao construto envolvimento. Em adição, foi dada ênfase à capacidade de agência dos atores da cúpula estratégica em moldar o contexto em termos de favorabilidade percebida à apresentação de propostas (issueselling) da média gerência em relação à renovação e ao desenvolvimento de novas capacidades.

É preciso destacar, ainda, o papel paradoxal da incerteza ambiental no modelo das capacidades dinâmicas a partir dos rituais de interação. Por um lado, a incerteza ambiental gera preocupações e necessidades de maiores e diferentes informações, o que contribui para que a cúpula estratégica aumente a favorabilidade do contexto e a abertura a novas propostas estratégicas de reconfiguração de ativos. Por outro lado, a incerteza opera no sentido oposto na relação direta com os gerentes de nível médio. Assim, a incerteza ambiental aumenta o risco percebido dos gerentes em propor ideias à alta gerência. Gerentes acreditam que, em tempos de incerteza, é arriscado fazer sugestões durante as conversações estratégicas que dão corpo aos rituais organizacionais de interação (Duttonet al., 1997).

Esse paradoxo gera forças contrárias no que concerne à influência no processo de desenvolvimento de novas capacidades organizacionais que exigirão competências gerenciais 
diferenciadas da alta gerência em ambientes mais turbulentos. Tal assertiva abre espaços para a exploração dessas relações à luz da influência de outras variáveis, bem como estimula a realização de estudos empíricos.

Assim, as proposições expostas anteriormente apontam que o conhecimento pode ser integrado, por meio de uma rede de relacionamentos e práticas discursivas e ritualísticas (Goffman, 1967; Collins, 2004) que permitam a ampliação das tradicionais fronteiras hierárquicas do processo estratégico. Essas redes intraorganizacionais fornecem mecanismos eficientes para a aquisição de novos conhecimentos, principalmente em ambientes altamente mutáveis, em que a rapidez e o escopo da integração de informações são considerados parâmetros para a contínua renovação das fontes da vantagem competitiva (Eisenhardt e Santos, 2006).

\section{Referências}

AMBROSINI, V.; BOWMAN, C. What are dynamic capabilities and are they a useful construct in strategic management? International Journal of Management Reviews, v. 11, n. 1, p. 29-49, 2009.

AUGIER, M.; TEECE, D.J. Strategy as evolution with design: the foundations of dynamic capabilities and the role of managers in the economic system. Organization Studies, v. 29, n. 8-9, p. 1187-1208, 2008.

BARNEY, J.B. Gaining and sustaining competitive advantage. Massachusetts: Addison-Wesley Publishing Company, 1996.

BARNEY, J.B. Organizational culture: can it be a source of sustained competitive advantage? Academy of Management Review, v. 11, n. 3, p. 656-665, 1986.

BARNEY, J.B.; CLARK, D.N. Resource-based theory. New York: Oxford University Press, 2007.

BURT, R.S. Structural holes: the social structure of competition. Cambridge: Harvard University Press, 1992.

COLLINS, R. Interaction ritual chains. Oxford: Princeton University Press, 2004.

COLLIS, D.J. Research note: how valuable are organizational capabilities? Strategic Management Journal, v. 15, p. 143-152, 1994.

DAVIS, J.P.; EISENHARDT, K.M.; BINGHAM, C.B. Optimal structure, market dynamism, and the strategy of simple rules. Administrative Science Quarterly, v. 54, n. 3, p. 413-452, 2009.

DOMENICO, M.L.D.; PHILLIPS, N. Sustaining de ivory tower: oxbridge formal dining as organizational ritual. Journal of Management Inquiry, v. 18, n. 4, p. 326-343, 2009.

DUTTON, J.E. et al. Reading the wind: how middle managers assess the context for selling issues to top managers. Strategic Management Journal, v. 18, n. 5, p. 407-425, 1997.

EISENHARDT, K.; MARTIN, J. Dynamic capabilities: what are they? Strategic Management Journal, v. 21 , n. 10, p. 1105-1121, 2000. 
EISENHARDT, K.M.; SANTOS, F.M. Knowledge-based view of the firm: a new theory of strategy? In: PETIGREW. A.; THOMAS, H.; WHITTINGTON, R. (Ed.). Handbook of Strategy and Management. Sage, 2006. p. 139-164.

GRANT, R.M. Toward a knowledge-based theory of the firm. Strategic Management Journal, v. 17, p. 109-122, 1996.

GOFFMAN, E. Interaction ritual. Doubleday: New York, 1967.

GOSS, D. Enterprise ritual: a theory of entrepreneurial emotion and exchange. British Journal of Management, v. 19, p. 120-137, 2008.

HELFAT, C.E. Guest editor's introduction to the special issue: the evolution of firm capabilities. Strategic Management Journal, v. 21, n. 10, p. 955-959, 2000.

HELFAT, C.E. Know-how and asset complementarity and dynamic capability accumulation: the case of R\&D. Strategic Management Journal, v. 18, n. 5, p. 339-360, 1997.

JOHNSON, L.D.; NEAVE, E.H.; PAZDERKA, B. Knowledge, innovation and share value. International Journal of Management Reviews, v. 4, n. 2, p. 101-134, 2002.

KOGUT, B.; ZANDER, U. Knowledge of the firm, combinative capabilities, and the replication of technology. Organization Science, v. 3, n. 3, p. 383-297, 1992.

LOCKETT, A.; THOMPSON, S.; MORGENSTERN, U. The development of the resource-based view of the firm: a critical appraisal. International Journal of Management Reviews, v. 11, n. 1, p. 9-28, 2009.

MARIOTTO, F.L. Mobilizando estratégias emergentes. Revista de Administração de Empresas, v. 43, n. 2, p. 78-93, 2003.

MILES, R.E.; SNOW, C.C. Organizational strategy, structure, and process. Stanford: Stanford University Press, 2003.

NODA, T.; BOWER, J.L. Strategy making as iterated processes of resource allocation. Strategic Management Journal, v. 17, n. s1, p. 159-192, 1996.

PENROSE, E.T. The theory of growth of the firm. London: Basil Blackwell, 1959.

RANSON, S.; HINNINGS, B.; GREENWOOD, R. The structuring of organizational structures. Administrative Science Quarterly, v. 25, n. 1, p.1-17, 1980.

SIRMON, D.G.; HITT, M.A. Contingencies within dynamic managerial capabilities: interdependent effects of resource investment and deployment on firm performance. Strategic Management Journal, v. 30, n. 13, p. 1375-1394, 2009.

SIRMON, D.G.; HITT, M.A.; IRELAND, R.D. Managing firm resources in dynamic environments to create value: looking inside the black box. Academy of Management Review, v. 32, n. 1, p. 273-292, 2007. 
TEECE, D.J. Explicating dynamic capabilities: the nature and microfoundations of (sustainable) enterprise performance. Strategic Management Journal, v. 28, n. 13, p. 1319-1350, 2007.

TEECE, D.J.; PISANO, G.; SCHUEN, A. Dynamic capabilities and strategic management. Strategic Management Journal, v. 18, n. 7, p. 509-533, 1997.

THOMPSON, J.D. Organizations in action. New York: McGraw-Hill, 1967.

WANG, C.L.; AHMED, P.K. Dynamic capabilities: a review and research agenda. International Journal of Management Reviews, v. 9, n. 1, p. 31-51, 2007.

WASSERMAN, S.; FAUST, K. Social network analysis. Cambridge: Cambridge University Press, 1999.

WINTER, S. Understanding dynamic capabilities. Strategic Management Journal, v. 24, p. 991-995, 2003.

WESTLEY, F.R. Middle managers and strategy: microdynamics of inclusion. Strategic Management Journal, v. 11, p. 337-351, 1990.

WOOLDRIDGE, B.; FLOYD, S.W. The strategy process, middle management involvement, and organizational performance. Strategic Management Journal, v. 11, p. 231-241, 1990.

WOOLDRIDGE, B.; FLOYD, S.W. Research notes and communications strategic process effects on consensus. Strategic Management Journal, v. 10, p. 295-302, 1989.

ZAHRA, S.A.; SAPIENZA, H.J.; DAVIDSON, P. Entrepreneurship and dynamic capabilities: a review, model and research agenda. Journal of Management Studies, v. 43, n. 4, p. 917-955, 2006.

ZOLLO, M.; WINTER, S. Deliberate learning and the evolution of dynamic capabilities. Organization Science, v. 13, p. 339-351, 2002.

Cristiano de Oliveira Maciel é professor no Programa de Pós-Graduação em Administração da Pontifícia Universidade Católica do Paraná (PUC-PR). E-mail: cristiano@pucpr.br.

Kawana Harue Sato é mestranda no Programa de Pós-Graduação em Administração da PUC-PR. E-mail: kawana.sato@gmail.com.

Heitor Takashi Kato é professor no Programa de Pós-Graduação em Administração da PUC-PR. E-mail: heitor.kato@pucpr.br. 